\title{
The answer is cilia, whatever the question may be!
}

\author{
Andrew Bush ${ }^{1,2,3}$, Claire Hogg ${ }^{3,4}$ \\ ${ }^{1}$ Head of Section (Paediatrics), Imperial College, London, UK; ${ }^{2}$ National Heart and Lung Institute, London, UK; ${ }^{3}$ Departments of Paediatrics and \\ Paediatric Respiratory Medicine, NHLI at Imperial College, London, UK; ${ }^{4}$ National PCD Diagnostic Service, Royal Brompton Hospital, London, UK \\ Correspondence to: Andrew Bush, MB, BS (Hons), MA, MD, FHEA, FRCP, FRCPCH, FERS, FAPSR, ATSF. Department of Paediatric Respiratory \\ Medicine, Royal Brompton Hospital, Sydney Street, London SW3 6NP, UK. Email: a.bush@imperial.ac.uk. \\ Comment on: Stewart E, Adams PS, Tian X, et al. Airway ciliary dysfunction: Association with adverse postoperative outcomes in nonheterotaxy \\ congenital heart disease patients. J Thorac Cardiovasc Surg 2018;155:755-63.e7.
}

Submitted Sep 06, 2018. Accepted for publication Sep 13, 2018.

doi: 10.21037/atm.2018.09.39

View this article at: http://dx.doi.org/10.21037/atm.2018.09.39

Once upon a time, as all the best children's stories start, life was very simple. Kartagener described a syndrome of sinusitis, bronchiectasis and situs inversus, and gave his name to the syndrome. Decades later, Bjorn Afzelius astutely noted the association between ultrastructural abnormalities in the sperm tails harvested from infertile males with situs inversus, and suggested that ciliary abnormalities were the underlying cause of Kartagener's syndrome. It also became clear that situs inversus was not an essential component to Kartagener's syndrome, and that approximately half of these patients had normal organ arrangement. Thus (contravening the wise advice of the late, great Dr Richard Asher, which was never to conflate clinical and pathological definitions), Kartagener's eponymous clinical syndrome was transmogrified into 'immotile cilia syndrome', and, via other nomenclatures, primary ciliary dyskinesia (PCD).

Subsequent advances in the understanding of ciliary biology led to the delineation of three apparently distinct types of cilia: (I) primary, non-motile cilia (confusingly, not the ciliary abnormality causing PCD); (II) nodal cilia, which determine foetal left-right orientation; and (III) motile cilia, which are responsible for moving the mucus layer across epithelial surfaces, or (less relevantly to the purposes of this annotation) propelling the unicellular algae chlamydomonas through liquid (1). It became apparent that mutations in multiple genes for primary cilia caused complex multisystem syndromes, characterised by diverse manifestations including retinopathy; kidney, liver, and pancreatic fibrocystic disease; anosmia; neurological abnormalities including ataxia; skeletal dysplasias and obesity, all apparently a far cry from Kartagener's syndrome. However, disorders of laterality and chronic respiratory symptoms are a feature of some primary ciliopathies, hinting at closer links than might have been thought.

However, the apparent clear blue water between classical Kartagener's syndrome and the primary ciliopathy syndromes has become muddied. Firstly, a case series demonstrated that complex congenital heart disease (CHD), especially with disorders of laterality, was a feature of Kartagener's syndrome, and this was subsequently confirmed by much larger series (2,3). Secondly, cases of retinitis pigmentosa (a well-known manifestation of the primary ciliopathies) was associated with $\mathrm{PCD}$, and the underlying link was mutations in the $R P G R$ gene. Next, it became clear that at least some primary ciliopathies have a PCD-like respiratory phenotype. Bardet Biedl syndrome is a non-motile ciliopathy characterised by features which include vision loss, obesity, polydactyly, learning disability and genital abnormalities. Forty-six patients with BardetBiedl syndrome (24 male, mean age 22 years) were evaluated for respiratory disease (4); $12 \%$ had unexplained respiratory distress at birth, $21 \%$ had been given a diagnosis of 'asthma' (presumably some form of lower airway disease), 33\% had otitis media, and $36 \%$ had persistent rhinitis. Ciliary beat frequency $(\mathrm{CBF})$ was intermediate between normal and $\mathrm{PCD}$, and nasal nitric oxide (nNO) measurements were mainly normal. There were also structural abnormalities (long, whip-like cilia and bulbous tips) (5), also found in other ciliopathies including Sensenbrenner and Joubert syndromes (unpublished). Alstrom's syndrome is another non-motile ciliopathy characterised by retinal degeneration, sensorineural hearing loss, obesity, insulin-resistant diabetes, hypertriglyceridemia, cardiomyopathy, hepatorenal disease and recurrent respiratory tract infections. In a series 
of 38 patients (6), 92\% recurrent otitis media, $50 \%$ had tympanostomy tubes placed, $61 \%$ had recurrent respiratory tract infections, and $50 \%$ sinusitis. Heterotaxy was not seen, and $\mathrm{nNO}$ was for the most part normal. Patients with primary ciliopathies characterised by retinal degeneration, principally Usher's syndrome, are variously reported to have abnormal CBF and reduced nNO (7). Study of nonmotile ciliopathies have also led to the expansion of the PCD clinical phenotype; whereas it was thought that the hearing loss in PCD was invariably due to chronic secretory otitis media, it is now recognised that sensorineural hearing loss is present in around $30 \%$ of PCD patients, related to abnormal function of cilia in otoliths (8). Finally, in a study of 35 children with CHD, ten were found to have abnormal ciliary motility. They underwent cranial ultrasound and brain magnetic resonance imaging pre- and postoperatively. Abnormal ciliary motility was associated with increased extra-axial cerebrospinal fluid $(\mathrm{CSF})(\mathrm{P}<0.001)$, belayed brain maturation $(\mathrm{P}<0.05)$ and subtle dysplasias (hippocampus, $\mathrm{P}<0.0078$; olfactory bulb $(\mathrm{P}<0.034)$, and composite dysplasia score $(\mathrm{P}<0.001)(9)$, again blurring the boundaries between the non-motile ciliopathies and PCD.

The range of diagnostic tests for PCD has also progressed, from standard transmission electron microscopy (TEM) which was once considered the 'gold standard'. The ever-increasing sophistication of testing has led to the development of risk scores to target investigations appropriately; key red flags include disorders of laterality, unexplained neonatal respiratory distress especially with lobar or segmental consolidation and prolonged oxygen dependency, and year-round daily rhinitis and wet cough $(10,11)$. The timing and order of diagnostic tests will depend on local availability. Key is to distinguish primary disease from secondary changes related to, for example, viral infections, thus avoiding over-diagnosing PCD. Algorhythms combining these tests have been proposed, in order to clarify how to make a diagnosis of PCD $(12,13)$.

The simplest first investigation in current usage is measurement of nNO, which is usually (but not invariably) very low (14). It should be noted, however, that other diseases are associated with a low $\mathrm{nNO}$, for example, cystic fibrosis, so further confirmatory testing is mandatory. Furthermore, cases of PCD with an elevated $\mathrm{nNO}$ are well described (15), so, if clinical suspicion is high, further testing should be pursued.

There are trans-Atlantic differences in the next steps to be undertaken. In Europe, measurement of CBF and function using high-speed video microscopy (HSVM) is the next step. Abnormalities may be the result of secondary changes, and must be interpreted with caution. Furthermore, PCD patients may have only very subtle abnormalities which are easily missed by those less experienced in the art of assessing ciliary beat patterns, although ciliary immotility is very obvious. Despite these caveats HSVM continues to be the most consistently abnormal test in patients subsequently diagnosed with PCD, and on many occasions its use will lead to the further diagnostic testing in more atypical cases.

Standard TEM was once thought to be the gold standard diagnostic test. Classical abnormalities, such as absence of the dynein arms are diagnostic, but others, such as transposition defects, may be secondary changes. If there is any doubt, a repeat sample should be obtained, or ciliary culture carried out (below). Also, proven diseasecausing genetic mutations in, for example, DNAH11, have no discernible TEM abnormalities. Electron microscopy has been taken to a new level of sophistication with the use of tomography, which has been used to detect subtle abnormalities missed by standard TEM in DNAH11 and bydin mutations, for example $(16,17)$.

Genetic testing is increasingly being used to diagnose, and commercial panels are available. The finding of two known disease-causing mutations in-trans is of course diagnostic. However, in addition to the usual problem of differentiating disease-causing mutations from single nucleotide polymorphisms of no clinical consequence, there are several hundred potential structural ciliary genes in which mutations could potentially cause $\mathrm{PCD}$, and cases of PCD with normal ciliary structural protein genes but abnormalities in genes encoding proteins involved in ciliary assembly have been described (18). This gives rise to an interesting line of speculation; could some children with a clinical cystic fibrosis phenotype of cystic fibrosis, but no diagnostic genotype, have mutations in a gene encoding for one of the many proteins involved in the transport of cystic fibrosis transmembrane regulator protein to the cell surface

A novel approach, which does not require sophisticated equipment, is immunofluorescence of ciliary proteins (19). This approach offers economies over genetic testing, since each ciliary protein may be absent as a result of multiple gene mutations. We stained for DNAH5 (an outer dynein arm heavy chain); DNALI1 (an inner dynein arm light chain); GAS8 (part of the nexin-dynein regulatory complex); and RSPH4A, RSPH9, and RSPH1 (all components of the radial spoke), thus covering the four key ultrastructural abnormalities seen in PCD. This antibody 
panel had excellent sensitivity and specificity in discovery and validation cohorts. The development of reliable new antibodies for DNAH11 and IFT8 will greatly enhance the utility of clinical testing for patients with normal TEM such as DNAH11 mutations and those with intraflagellar transport defects (more commonly seen in the syndromic ciliopathies such as Jeune's asphyxiating thoracic dystrophy).

Differentiating PCD from secondary ciliary dyskinesia is a key issue, and chronic infective rhinitis can certainly cause secondary abnormalities of $\mathrm{CBF}$ and structure. Culture of ciliated epithelium may be utilised to eliminate the effects of infection and restore ciliary function in vitro for functional and structural testing (20). This is a challenging test, and is not needed if a clear-cut diagnosis has been established by less demanding tests, but if there is diagnostic doubt, then ciliary culture is indicated.

The above sets in context a recent manuscript describing abnormal tests of ciliary function in 63 infants with nonheterotaxic CHD (although surprising, a patient with complete mirror image arrangement was included, this will not have affected the conclusions of the manuscript) (21). Interestingly, these cardiac lesions have been reported in nonmotile ciliopathies, although there is no suggestion that this was a factor in this series. The authors studied ciliary function by measuring $\mathrm{CBF}$ and $\mathrm{nNO}$, two mainstream tests of ciliary function. Previously, one or both of these same tests in an out-patient study had been abnormal in $33 \%$ of heterotaxic and $17 \%$ of non-heterotaxic patients with CHD (22) In the present study, the authors hypothesised that nonheterotaxic patients with CHD with evidence of impaired ciliary function demonstrated on these tests would have increased post-operative morbidity and more respiratory complications. Generally, outcomes were excellent, with only one peri-operative death despite the severity and complexity of many of the lesions. The prevalence of abnormal ciliary function tests was higher than previously reported (32\% abnormal CBF, 39\% abnormal nNO, $6 \%$ both, meaning an astonishing two thirds of the patients had an abnormality of ciliary function; this proportion may have risen still further if more sophisticated ciliary studies had been performed). Those with abnormal CBF were more likely to require non-invasive ventilation, have more viral infections and be prescribed respiratory medication, whereas those with low nNO were more likely to acquire bacterial infections, and interestingly, had worse pre- and post-operative systolic ventricular function at the time of surgery, but not at the time of study.

This is an important study, albeit with two methodological caveats. CBF is not an easy technique, and the manuscript would have been strengthened by a normal control population. The evaluation of the samples by three different, blinded investigators is a strength, but it is a pity CBF was not quantitated, and only given a qualitative score. Also, reproducibility data over time in the same patients would have been of interest. Although there is no obvious biological reason why $\mathrm{CBF}$ and $\mathrm{nNO}$ in non-heterotaxic $\mathrm{CHD}$ should vary over time, confirmation would have been valuable, and perhaps both these points could be addressed in future studies. In this context, a recent paper has highlighted that respiratory tract infections can cause a sharp drop in $\mathrm{nNO}$ in infants (23).

As the authors point out, there are at least two potential mechanisms whereby ciliary dysfunction could lead to worse post-operative outcomes, namely reduced mucociliary clearance (MCC) and impairment of host immunity. It would be important to try to determine the pathway, because each might be susceptible to an intervention, which would of course need to be tested. It is unclear from the current manuscript as to whether there is a readout in terms of in vivo MCC from the CBF abnormalities described. This would readily be testable with radioisotope clearance studies. If this was the case, then treating these patients with nebulised hypertonic saline, which is known to increase MCC in a dose dependent fashion (24) before and over the high-risk peri-operative period would be the obvious step to trial. It would be worth considering a different antibiotic strategy if immunodeficiency were to be the issue.

The association of low $\mathrm{nNO}$ and worse ventricular function is also challenging to interpret. Nitric oxide is a powerful and selective pulmonary vasodilator, but production by endothelial nitric oxide synthase (eNOS), which is likely normal in PCD (25), is most relevant. It is entirely speculative, but could endothelial dysfunction in some way have contributed to impaired cardiac function? The observation needs to be confirmed in another group, but could possibly open up important therapeutic avenues.

Also, curious, and not discussed by the authors, is that more than $10 \%$ had abnormalities of the kidneys and urinary tract, of whom all had at least one abnormality of ciliary function. We know that adult and paediatric polycystic kidney disease, nephronophthisis and renal dysplasia are all manifestations of ciliopathy (1), but could this series be hinting at a wider renal and urinary phenotype? Further work is needed to address this possibility; genetic testing would play a useful role here with increasingly comprehensive ciliopathy gene panels and 
next generation sequencing now available.

As with all the best studies, the current investigation raises more questions than answers about the relationship between cilia and CHD, both heterotaxic and nonheterotaxic. It is a truth universally acknowledged that the tertiary specialist may be blind to paediatric disease not immediately central to their speciality; indeed, to be in a specialist clinic with an illness belonging to a different speciality is a very perilous situation! All children cough and have rhinitis from time to time, and many is the child languishing in a cardiac clinic with complex CHD and heterotaxy who a 'few more snuffles than average' turns out to have full-blown PCD; and it is not to be wondered that such children have more operative complications. But what of the child who falls way short of a PCD diagnosis by conventional criteria, yet has some evidence of ciliary dysfunction? Do these children have a mild, atypical form of PCD, rather akin to atypical cystic fibrosis, in which conventional tests may also be normal or equivocal? Widening the definition of such cases to include them within a group of respiratory ciliopathies may be timely, with PCD at the extreme end of a spectrum that includes an array of other conditions with an, as yet undefined but likely, underlying association with ciliary defects. On a practical level, should all CHD patients have ciliary function studies pre-operatively to identify a high-risk group? Measurement of CBF in all would be resource intensive, but nNO is quick, cheap and easy to measure. Of course, this would only be worth doing if an intervention improved outcome, and this has yet to be shown. Whatever the answer to these questions, it is clear that, despite numerous papers, we still have a lot to learn about cilia and ciliopathy. But we should not neglect what we know already during the learning process; cardiologists and cardiac surgeons, if you see a child with complex CHD and heterotaxy, or CHD and any of the key features of PCD—neonatal onset of rhinitis and respiratory distress, yearlong wet cough and rhinitis, recurrent respiratory infections-have a very low threshold for referral for exclusion of PCD, preferably before surgery. At the very least consider the diagnosis and ask your physiotherapist to assess the child preoperatively and institute airway clearance techniques if indicated.

\section{Acknowledgements}

A Bush is an NIHR Senior Investigator and additionally was supported by the NIHR Respiratory Disease Biomedical Research Unit at the Royal Brompton and Harefield NHS
Foundation Trust and Imperial College London.

\section{Footnote}

Conflicts of Interest: The authors have no conflicts of interest to declare.

\section{References}

1. Dehlink E, Hogg C, Carr S, et al. Clinical phenotype and current diagnostic criteria for Primary Ciliary Dyskinesia. Expert Rev Respir Med 2016:19:1-13.

2. Kennedy MP, Omran H, Leigh MW, et al. Congenital heart disease and other heterotaxic defects in a large cohort of patients with primary ciliary dyskinesia. Circulation 2007;115:2814-21.

3. Best S, Shoemark A, Rubbo B, et al. Risk factors for situs defects and congenital heart disease in primary ciliary dyskinesia. Thorax 2018. [Epub ahead of print].

4. Shoemark A, Dixon M, Beales PL, et al. Bardet Biedl syndrome: motile ciliary phenotype. Chest 2015;147:764-70.

5. Shah AS, Farmen SL, Moninger TO, et al. Loss of BardetBiedl syndrome proteins alters the morphology and function of motile cilia in airway epithelia. Proc Natl Acad Sci U S A 2008;105:3380-5.

6. Boerwinkle C, Marshall JD, Bryant J, et al. Respiratory manifestations in 38 patients with Alström syndrome. Pediatr Pulmonol 2017;52:487-93.

7. Heffler E, Marchese C, Boita M, et al. Nasal nitric oxide in patients with inherited retinal dystrophies. J Investig Med 2015;63:554-7.

8. Kreicher KL, Schopper HK, Naik AN, et al. Hearing loss in children with primary ciliary dyskinesia. Int J Pediatr Otorhinolaryngol 2018;104:161-5.

9. Panigrahy A, Lee V, Ceschin R, et al. Brain Dysplasia Associated with Ciliary Dysfunction in Infants with Congenital Heart Disease. J Pediatr 2016;178:141-8.e1.

10. Behan L, Dimitrov BD, Kuehni CE, et al. PICADAR: a diagnostic predictive tool for primary ciliary dyskinesia. Eur Respir J 2016;47:1103-12.

11. Leigh MW, Ferkol TW, Davis SD, et al. Clinical Features and Associated Likelihood of Primary Ciliary Dyskinesia in Children and Adolescents. Ann Am Thorac Soc 2016;13:1305-13.

12. Lucas JS, Barbato A, Collins SA, et al. European Respiratory Society guidelines for the diagnosis of primary ciliary dyskinesia. Eur Respir J 2017;49. 
13. Shapiro AJ, Davis SD, Polineni D, et al; American Thoracic Society Assembly on Pediatrics. Diagnosis of Primary Ciliary Dyskinesia. An Official American Thoracic Society Clinical Practice Guideline. Am J Respir Crit Care Med 2018;197:e24-39.

14. Shapiro AJ, Josephson M, Rosenfeld M, et al. Accuracy of Nasal Nitric Oxide Measurement as a Diagnostic Test for Primary Ciliary Dyskinesia. A Systematic Review and Meta-analysis. Ann Am Thorac Soc 2017;14:1184-96.

15. Shoemark A, Moya E, Hirst RA, et al. High prevalence of CCDC103 p.His154Pro mutation causing primary ciliary dyskinesia disrupts protein oligomerisation and is associated with normal diagnostic investigations. Thorax 2018;73:157-66.

16. Olbrich H, Schmidts M, Werner C, et al. Recessive HYDIN mutations cause primary ciliary dyskinesia without randomization of left-right body asymmetry. Am J Hum Genet 2012;91:672-84.

17. Shoemark A, Burgoyne T, Kwan R, et al C. Primary ciliary dyskinesia with normal ultrastructure: three-dimensional tomography detects absence of DNAH11. Eur Respir J $2018 ; 51$.

18. Olcese C, Patel MP, Shoemark A, et al. X-linked primary ciliary dyskinesia due to mutations in the cytoplasmic axonemal dynein assembly factor PIH1D3. Nat Commun

Cite this article as: Bush A, Hogg C. The answer is cilia, whatever the question may be! Ann Transl Med 2018;6(Suppl 1):S32. doi: 10.21037/atm.2018.09.39
2017;8:14279.

19. Shoemark A, Frost E, Dixon M, et al. Accuracy of Immunofluorescence in the Diagnosis of Primary Ciliary Dyskinesia. Am J Respir Crit Care Med 2017;196:94-101

20. Hirst RA, Rutman A, Williams G, et al. Ciliated air-liquid cultures as an aid to diagnostic testing of primary ciliary dyskinesia. Chest 2010;138:1441-7.

21. Stewart E, Adams PS, Tian X, et al. Airway ciliary dysfunction: Association with adverse postoperative outcomes in non-heterotaxy congenital heart disease patients. J Thorac Cardiovasc Surg 2018;155:755-63.e7.

22. Garrod AS, Zahid M, Tian X, et al. Airway ciliary dysfunction and sinopulmonary symptoms in patients with congenital heart disease. Ann Am Thorac Soc 2014;11:1426-32.

23. Marthin JK, Philipsen MC, Rosthoj S, et al. Infant nasal nitric oxide over time: natural evolution and impact of respiratory tract infection. Eur Respir J 2018;51.

24. Robinson M, Hemming AL, Regnis JA, et al. Effect of increasing doses of hypertonic saline on mucociliary clearance in patients with cystic fibrosis. Thorax 1997;52:900-3.

25. Suri R, Paraskakis E, Bush A. Alveolar, but not bronchial nitric oxide production is elevated in cystic fibrosis. Pediatr Pulmonol 2007;42:1215-21. 